\title{
Spectral synthesis of stellar populations
}

\author{
J. M. Gomes ${ }^{1}$, R. Cid Fernandes ${ }^{1}$ \\ and R. Rodrigues Lacerda ${ }^{1}$ \\ ${ }^{1}$ Universidade Federal de Santa Catarina, Florianópolis, SC, Brazil
}

\begin{abstract}
We present a new method to decompose the stellar population mixtures in galaxies. The method combines the spectra of simple stellar populations (SSP) of different ages and metallicities, computed with the recently published high spectral resolution evolutionary synthesis models of Bruzual \& Charlot (2003). Statistical mechanics techniques, based on Markov Chain Monte Carlo methods, are used in the search for the combination of SSPs which best matches an observed spectrum. The spectral base covers 10 ages and 3 metallicities. The method provides the Star Formation History (SFH) expressed in a population vector $\vec{x}$, whose components represent the fractional contribution of each SSP to the total flux (or mass). The code also estimates the velocity dispersion $\sigma_{\star}$. A series of simulations were performed to evaluate the code's ability to recover the input parameters (SFH, extinction and velocity dispersion), to investigate the effects of noise in the data and map intrinsic degeneracies of stellar populations.
\end{abstract}

\section{Introduction}

The new generation of high spectral resolution evolutionary synthesis models offers the interesting possibility of fitting the whole spectra of a galaxy, instead of just its colors and some spectral indices. We have developed and tested one such spectral synthesis method, which searches for the linear combination of $N_{\star}$ Simple Stellar Populations (SSP) which best fits a given observed spectrum $O_{\lambda}$. The equation for a model spectrum $M_{\lambda}$ is

$$
M_{\lambda}\left(\vec{x}, A_{V}, v_{\star}, \sigma_{\star}\right)=M_{\lambda_{0}}\left[\sum_{j=1}^{N_{\star}} b_{j, \lambda} r_{\lambda}\right] \otimes G\left(v_{\star}, \sigma_{\star}\right)
$$

where,

- $b_{j, \lambda} \equiv L_{\lambda}^{S S P}\left(t_{j}, Z_{j}\right) / L_{\lambda_{0}}^{S S P}\left(t_{j}, Z_{j}\right)$ is the spectrum of the j-th SSP normalized at $\lambda_{0}$. The $L_{\lambda}^{S S P}\left(t_{j}, Z_{j}\right)$ spectra are take from BC03 models.

- $r_{\lambda}=10^{-0.4\left(A_{\lambda}-A_{\lambda_{0}}\right)}$ is the reddening term. Extinction is modeled as due to an uniform dust screen, parametrized by $A_{V}$.

- $\vec{x}$ is the population vector, whose components $x_{j}$ represent the fractional contribution of each SSP in the base to the total synthetic flux at $\lambda_{0}$.

- $M_{\lambda_{0}}$ is the synthetic flux at the normalization wavelength.

- $G\left(v_{\star}, \sigma_{\star}\right)$ is the line-of-sight stellar velocity distribution.

We evaluate the match between the model $M_{\lambda}$ and observed $O_{\lambda}$ spectra by

$$
\chi^{2}\left(\vec{x}, A_{V}, v_{\star}, \sigma_{\star}\right)=\sum_{j=1}^{N_{\star}}\left[\left(O_{\lambda}-M_{\lambda}\right) \omega_{\lambda}\right]^{2}
$$

where $\omega_{\lambda}$ is the weight spectrum defined as the inverse of the noise in $O_{\lambda}$. Regions containing emission lines or spurious features are masked out by assigning $\omega_{\lambda}=0$. The search for the best parameters is carried out with a simulated annealing method. 

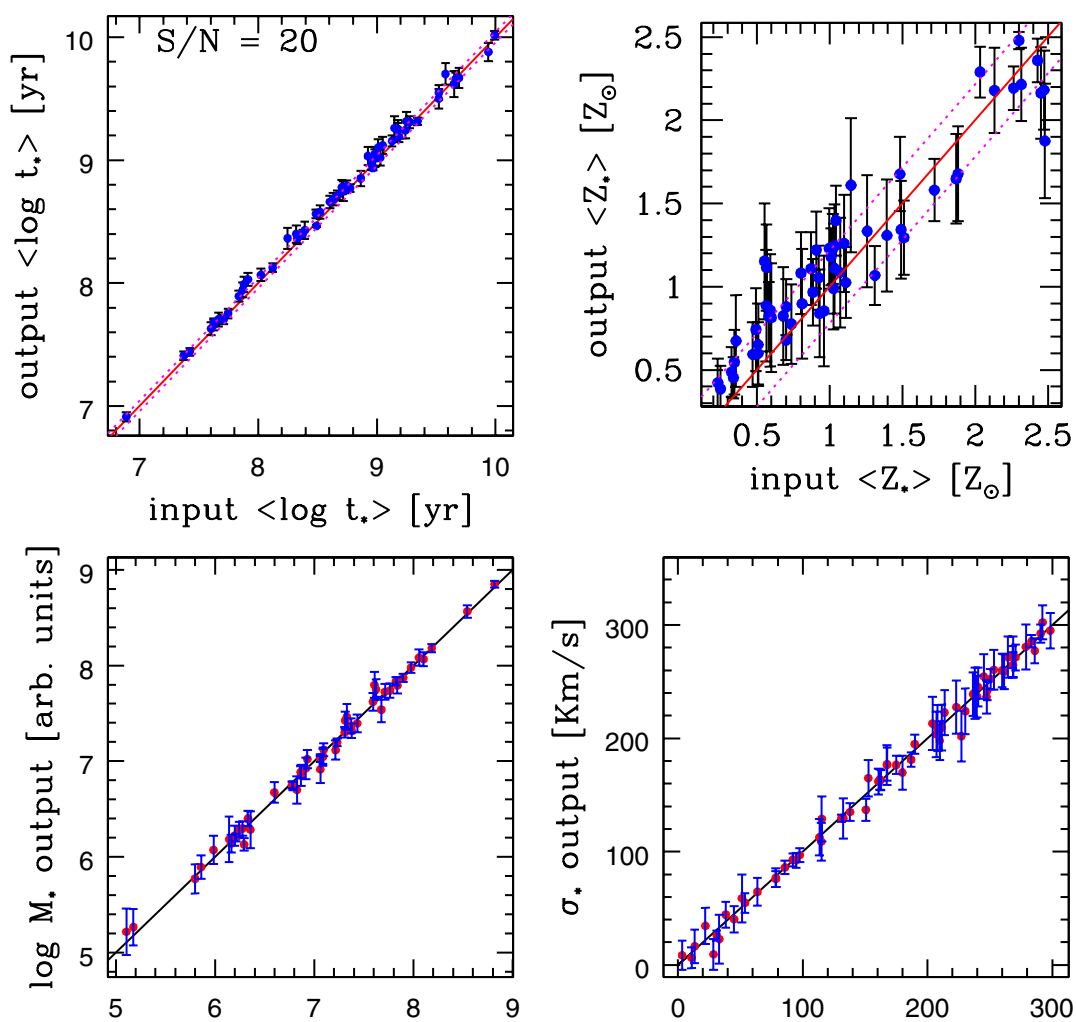

$\log \mathrm{M}_{*}$ input [arb. units]

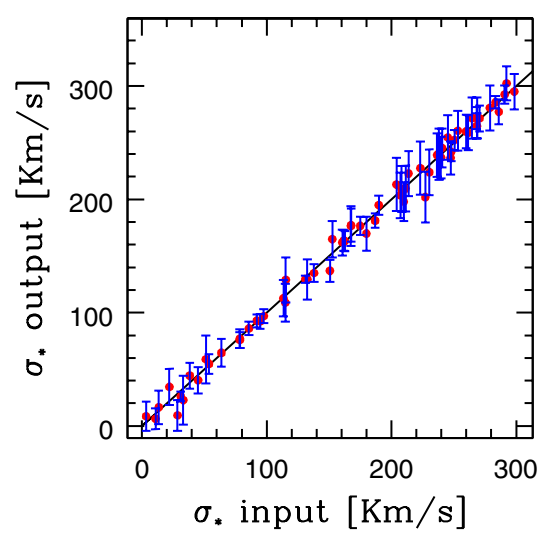

Figure 1. Output vs Input values. We are able to recover useful information on the mean stellar-metallicity $\left\langle Z_{\star}\right\rangle\left(=\sum_{j=1}^{N_{\star}} x_{j} Z_{j}\right)$ for $S / N=20$ or better. Other parameters such as mean stellar-age $<\log t_{\star}>\left(=\sum_{j=1}^{N_{\star}} x_{j} \log t_{j}\right)$ are well recovered for even lower S/N. The total stellar mass and velocity dispersion are also well recovered.

\section{Simulations and Conclusions}

We made tests with galaxies generated out of the base in order to evaluate the code's ability to recover input parameters $\left(\vec{x}, A_{V}, \sigma_{\star}, \ldots\right)$, to calibrate its technical parameters (number of steps, step-size, etc.) and to investigate the effects of noise in the data. For a $S / N=\infty$ the code recovers the input with excellent precision. Noise has the effect of inducing substantial rearrangements among $\vec{x}$ components corresponding to spectrally similar populations, so the base becomes linearly dependent. The solution in this case is to marginalize over the details and work with more robust quantities, such as mean stellar age, mean stellar metallicities, etc. We can see an important result of these simulations in Figure 1: the mean stellar metallicity is well recovered for a $S / N=20$ or better.

\section{Acknowledgements}

We would like to thanks the financial support of CNPq.

\section{References}

Bruzual, G., \& Charlot, S. 2003, MNRAS, 344, 1000 